\title{
BMJ open A possible association between a dysfunctional skin barrier (filaggrin null-mutation status) and diabetes: a cross-sectional study
}

\author{
Jacob P Thyssen, ${ }^{1}$ Allan Linneberg, ${ }^{2}$ Berit C Carlsen, ${ }^{1}$ Jeanne D Johansen, ${ }^{1}$ \\ Kåre Engkilde, ${ }^{1}$ Torben Hansen, ${ }^{3,4}$ Flemming Pociot, ${ }^{5}$ Oluf Pedersen, ${ }^{3,6,7}$ \\ Michael Meldgaard, ${ }^{8}$ Pal B Szecsi, ${ }^{8}$ Steen Stender, ${ }^{8}$ Torkil Menné ${ }^{1}$
}

To cite: Thyssen JP, Linneberg A, Carlsen BC, et al. A possible association between a dysfunctional skin barrier (filaggrin null-mutation status) and diabetes: a cross-sectional study. BMJ Open 2011;1: e000062. doi:10.1136/ bmjopen-2011-000062

- Prepublication history for this paper is available online. To view these files please visit the journal online (http:// bmjopen.bmj.com).

Received 10 January 2011 Accepted 7 February 2011

This final article is available for use under the terms of the Creative Commons Attribution Non-Commercial 2.0 Licence; see http://bmjopen.bmj.com
For numbered affiliations see end of article.

\section{Correspondence to} Dr Jacob Pontoppidan Thyssen;

jacpth01@geh.regionh.dk

\section{ABSTRACT}

Background: Filaggrin proteins are located in the skin and prevent epidermal water loss and impede the entry of micro-organisms, allergens and chemicals. Filaggrin null mutations are strongly associated with ichthyosis vulgaris and atopic dermatitis.

Objective: The authors aimed to investigate the association between filaggrin null mutations, atopic dermatitis and diabetes.

Design: A random sample of 3335 adults from the general population in Denmark was filaggringenotyped for R501X and 2282del4 null-mutations and questioned about atopic dermatitis and diabetes. Furthermore, two independent study populations of patients with type $1(n=104)$ or $2(n=774)$ diabetes were genotyped.

Results: In a crude data analysis, a positive association was detected between the filaggrin null genotype and, respectively, subjects from the general population who reported diabetes $(p=0.04)$ and patients with established type 2 diabetes $(p=0.073)$. Adjustment for age and gender resulted in significant associations for patients with type 2 diabetes $(p=0.048)$ and subjects with self-reported diabetes $(p=0.032)$.

Conclusions: Adult Danes with a filaggrin null genotype had a significantly increased prevalence of self-reported diabetes. This finding was replicated when an independent sample of Danish patients with established type 2 diabetes was compared with control subjects from the general population.

\section{INTRODUCTION}

The outermost part of the skin acts as a barrier that protects against damage following exposure to, for example, mechanical insults, UV light, extreme temperatures, chemicals and micro-organisms. Furthermore, the skin has neuroendocrine and immune functions. Filaggrin proteins are crucial components of the terminal differen-

\section{ARTICLE SUMMARY}

Article focus

- A few studies have suggested the existence of an inverse association between atopic dermatitis and type 1 diabetes.

- The existence of a specific endotype of asthma that is not driven by sensitisation but rather driven by skin barrier dysfunction was recently suggested.

- It is unknown whether a putative impairment of the skin barrier may increase the propensity to low-grade inflammation in other organs as well.

Key messages

- Data from a general population study suggested that the prevalence of filaggrin null mutations was higher in adult Danes who reported diabetes than in non-diabetics.

- This finding was replicated when an independent sample of Danish patients with type 2 diabetes was compared with participants from the general population who did not report diabetes and who had normal fasting plasma-glucose and glycated haemoglobin (HbA1c) levels.

Strengths and limitations of this study

- Two independent samples were investigated and showed similar results.

- The question on self-reported diabetes was not validated in the general population allowing for misclassification. 
with asthma and allergic rhinitis in combination with atopic dermatitis..$^{5} 6$

Few studies have investigated the skin barrier function in diabetics. An impaired state of stratum corneum hydration and a decreased skin surface lipid content in diabetic skin have been suggested in a mixed population of type 1 and 2 diabetics, and in diabetic mice. ${ }^{78} \mathrm{~A}$ case-control study found no difference in stratum corneum hydration and transepidermal water loss between diabetics and controls. ${ }^{9}$ A few studies have suggested the existence of an inverse association between atopic dermatitis and type 1 diabetes; a finding that may be explained by the T-helper (Th) cell 1 and 2 dichotomy. ${ }^{10}{ }^{11}$ To our knowledge, no studies so far have investigated the possible association between diabetes type 2 and atopic dermatitis.

Filaggrin genotyping was recently performed in 3335 adults from the general population in Copenhagen. ${ }^{3}$ In our data analyses, we noticed a relatively high frequency of the null genotype in subjects who reported diabetes. We therefore hypothesised that a putative impairment of the skin barrier increases the propensity to low-grade inflammation, which again, in concert with other factors, could increase the risk of diabetes. In favour of such a mechanism, Bønnelykke et al recently found a filaggrinassociated pattern of atopic diseases in early childhood characterised by early onset of atopic dermatitis, early onset of asthma (independent of atopic dermatitis status) with severe exacerbations and later development of sensitisation. ${ }^{12}$ Thus, the existence of a specific endotype of asthma that is driven not by sensitisation but rather by skin-barrier dysfunction was suggested, since filaggrin is not expressed in airway mucosa.

Here, we investigated whether an association could be found between self-reported diabetes and, respectively, atopic dermatitis and filaggrin null mutation status. We included two independent study populations of patients who had type 1 or 2 diabetes, to determine whether a possible association could be replicated.

\section{MATERIALS AND METHODS \\ Study populations \\ Ethic statement}

The Ethical Committee of Copenhagen County approved the study (KA-20060011). Written and verbal consent was given by the participants to be included in the study and for their information to be stored in the hospital database and used for research.

Three independent Danish populations were included in the current study: (1) a random sample of adults from the general population in Copenhagen ${ }^{13}$; (2) patients with type 1 diabetes $^{14}$; and (3) patients with type 2 diabetes.

\section{Adults from the general population}

Between June 2006 and May 2008, a cross-sectional study was performed in the general population in Copenhagen. A random sample of 7931 subjects aged
18-69 years was obtained from the Danish Central Personal Register. All were adults with Danish citizenship and born in Denmark. A total of 3471 (43.7\%) subjects participated in a general health examination, and 3335 (96.1\%) were filaggrin-genotyped for the 2282del4 and R501X mutations. The participation rate was higher among older age groups. ${ }^{15}$

\section{Patients with type 1 diabetes}

A total of 104 patients diagnosed as having type 1 diabetes between 1981 and 2004 were randomly selected from a large incident cohort. ${ }^{14}$ All patients were diagnosed before age 18 years and according to WHO criteria. They were all positive for protein tyrosine phosphatase-like protein and/or glutamic acid decarboxylase 65 antibodies at diagnosis $(0-3$ months prior to the first insulin injection).

\section{Patients with type 2 diabetes}

A total of 774 (299 women, 475 men; age $65.2 \pm 11.4$ years; BMI, $30.6 \pm 5.8 \mathrm{~kg} / \mathrm{m}^{2}$ ) unrelated patients diagnosed as having type 2 diabetes sampled randomly from the outpatient clinic at Steno Diabetes Center from 2005 to 2007 were included in the study.

\section{Measurements (general population only)}

Height and weight were measured in light indoor clothing and without shoes. Waist circumference was measured in the upright position midway between the iliac crest and the lower costal margin. Body mass index (BMI) was calculated in $\mathrm{kg} / \mathrm{m}^{2}$. Blood-pressure measurements were performed after 5 min rest in the sitting position with a mercury sphygmomanometer. If the systolic or diastolic blood pressure exceeded 140 and $90 \mathrm{~mm} \mathrm{Hg}$, respectively, repeated measurements were made later during the health examination with the participant in a lying position. The lowest value was used.

Blood samples were drawn after a $12 \mathrm{~h}$ overnight fast. Fasting plasma glucose was analysed by a glucose oxidase method (Hitachi 912 system, Roche Diagnostics, Mannheim, Germany). Glacated hemaglobin (HbAlc) was analysed by the HPLC method (TOSOH, Minato, Japan). Serum insulin was measured using the AutoDELFIA insulin kit (Perkin-Elmer/Wallac, Turku, Finland). Fasting concentrations of total-, HDL- and LDL cholesterol as well as triglyceride were measured using enzymatic colorimetric methods (Roche Molecular Biochemicals, Mannheim, Germany). ${ }^{16}$ The homeostasis model assessment of insulin resistance (HOMA-IR) was used to estimate the degree of insulin resistance. The HOMA-IR index was estimated from fasting plasma glucose and fasting serum insulin concentrations using the following formula: HOMA-IR index $=($ fasting plasma glucose $(\mathrm{mmol} / \mathrm{l}) \cdot$ fasting serum insulin $(\mathrm{mU} / \mathrm{l})) / 22.5 .{ }^{17}$

\section{Filaggrin genotyping}

Regions covering the mutations R501X and 2282del4 of the filaggrin gene were amplified from genomic DNA by PCR, and the obtained DNA fragments were hybridised 
to both mutation-specific and wildtype-specific probes attached to fluorescent micro beads (Luminex, Austin, Texas), and subsequently analysed on a BioPlex 200 (Biorad, Hercules, California). ${ }^{3}$

\section{Questionnaire (general population only)}

Participants from the cross-sectional general population study were sent a standard invitation letter and a questionnaire on health, lifestyle and socio-economic factors. One question addressed diabetes. An affirmative answer to the question 'Have you ever been told that you suffered from diabetes?' was used to identify subjects with diabetes. Thus, no questions were used that potentially could differentiate between subjects with type 1 and 2 diabetes.

A history of atopic dermatitis was defined by the UK Working Party's diagnostic criteria for atopic dermatitis as a history of an itchy skin condition plus a minimum of two of four minor criteria. ${ }^{18}$ The minor criteria were: (1) a history of involvement of the skin creases, (2) a personal history of asthma or hay fever, (3) a history of general dry skin in the last year and (4) onset before the age of 2 years.

\section{Statistical analysis}

Deviation from the Hardy-Weinberg equilibrium was tested using the free online calculator at the Online Encyclopedia for Genetic Epidemiology studies (http://www.oege.org/software/hwe-mr-calc.shtml) for both filaggrin null mutations. The filaggrin null genotype was defined as subjects who were either heterozygotic or homozygotic for mutations R501X or 2282del4.

Based on data from the general population study, three subgroups were constructed: a 'non-diabetes group' ( $\mathrm{n}=3136)$, a 'screen-detected diabetes group' $(n=66)$ and a 'self-reported diabetes group' $(n=133)$. Non-diabetics gave a negative answer to the question about diabetes and had a fasting glucose of $<7 \mathrm{mmol} / 1$ and HbA1c of $<6.5 \%$. Screen-detected diabetics did not report diabetes but had a fasting glucose $\geq 7 \mathrm{mmol} / 1$ $(n=58)$ and/or HbA1c $\geq 6.5 \% \quad(n=8)$. Finally, diabetics reported diabetes in the questionnaire. Two additional subgroups were constructed based on the two independent study populations of patients with diabetes, the type 1 diabetes group $(\mathrm{n}=104)$ and the type 2 diabetes group $(\mathrm{n}=774) \quad($ table 1$)$.

\section{Logistic regression analyses using data from the general} population study and from the cohorts of patients with type 1 and/or 2 diabetes (table 2)

A logistic regression model was performed with 'filaggrin null mutation status' as the dependent variable, and with gender, age and diabetes subgroup ('non-diabetes,' 'screen-detected diabetes,' 'self-reported diabetes,' 'type 1 diabetes' and 'type 2 diabetes') as the independent variables. In this model, a test for interaction between age and filaggrin mutation status was performed using a log-likelihood ratio test. This was done to test whether an association between filaggrin mutation status and diabetes status could depend on age. Another logistic regression analysis was performed with 'diabetes' as the dependent variable ('self-reported diabetics' and 'screen-detected diabetics' from the general population as well as patients from the 'type 2 diabetes group' were registered as diabetics, whereas patients with type 1 diabetes were regarded as missing data) and filaggrin mutation status ('wildtype,' 'null-mutation') and BMI $\left({ }^{\prime}<25\right.$, ' ' $25-30$, ' ' $>30$ ' $\left.\mathrm{kg} / \mathrm{m}^{2}\right)$ as the independent variables. In this analysis, an interaction term between filaggrin mutation status and BMI was inserted to determine whether the association between filaggrin mutations and diabetes depended significantly on BMI.

\section{Logistic regression analysis using data from the general population only (table 3 )}

'Self-reported atopic dermatitis' was used as the independent variable, and gender, age, diabetes subgroup ('non-diabetes,' 'screen-detected diabetes' and 'selfreported diabetes') and filaggrin mutation status ('wildtype,' 'null-mutation') were explanatory variables. In a similar regression analysis, a test for interaction between diabetes subgroup and filaggrin mutation status was performed using a log-likelihood ratio test. This was carried out to determine whether the association between atopic dermatitis and diabetes depended significantly on filaggrin mutation status.

Characteristics were compared using the $\chi^{2}$ test. Oneway ANOVA was used to compare means between different subgroups. Associations were expressed as ORs with 95\% CIs. Data analyses were performed using SPSS for Windows (release 15.0).

\section{RESULTS}

A total of 3335 participants from the general population study (55.3\% women), 104 patients diagnosed as having type 1 diabetes (40.4\% women) and 774 patients diagnosed as having type 2 diabetes (38.5\% women) were genotyped for the R501X and 2282del4 filaggrin null mutations. The observed genotype prevalences of both polymorphisms did not deviate significantly $(p>0.05)$ from the expected prevalences under the Hardy-Weinberg equilibrium assumption in any of these three populations.

The prevalence of self-reported diabetes was $4 \%$ in the general population (3.3\% in women and $4.9 \%$ in men) (table 1). The vast majority of participants who reported diabetes were expected to suffer from type 2 diabetes. To add evidence to this assumption, we calculated the HOMA-IR in subjects without diabetes, those with screen-detected diabetes and those with self-reported diabetes (table 1). One-way ANOVA analysis revealed a statistically significant difference in the HOMA-IR mean between the three subgroups $(\mathrm{p}<0.001)$. This supports the notion that type 2 diabetes was likely for the majority of cases with self-reported diabetes.

The prevalence of filaggrin mutations and atopic dermatitis was, respectively, $7.8 \%$ (95\% CI 7.0 to 8.8 ) and 


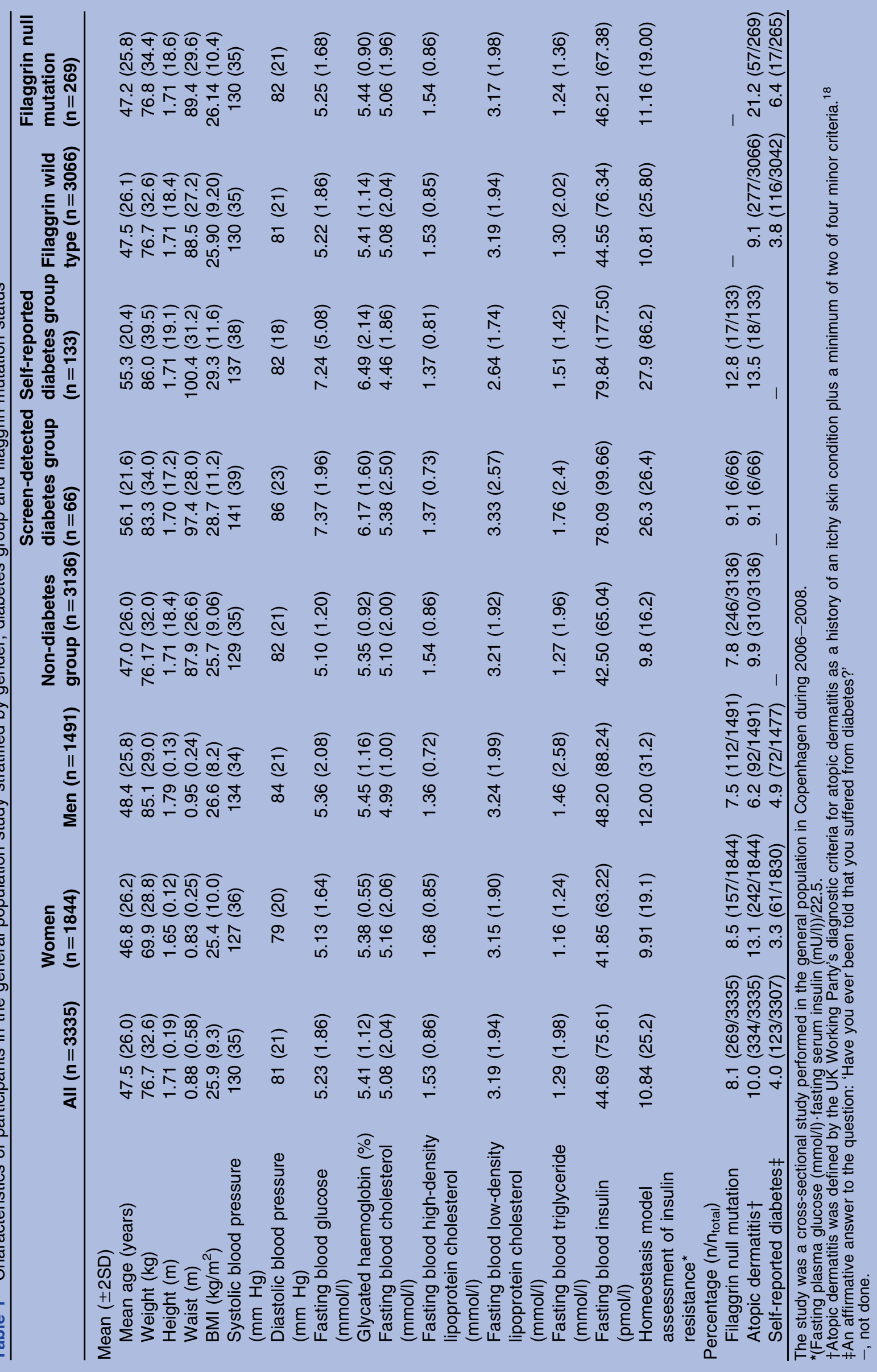


Table 2 Two logistic regression analyses with the outcome filaggrin null mutation status and diabetes, respectively, and adjusted for variables shown in the table as well as age

\begin{tabular}{|c|c|c|}
\hline \multirow[b]{3}{*}{ Explanatory variables } & \multicolumn{2}{|c|}{ General population, patients with type 1 and 2 diabetes $(n=4213)$} \\
\hline & \multicolumn{2}{|c|}{ Filaggrin null mutation } \\
\hline & Percentage $\left(\mathrm{n} / \mathrm{n}_{\text {total }}\right)$ & Adjusted OR* with $95 \% \mathrm{Cl}$ \\
\hline \multicolumn{3}{|l|}{ Sex } \\
\hline Men & $7.8(159 / 2029)$ & 1 (reference) \\
\hline Women & $8.8(193 / 2184)$ & $1.18(0.94$ to 1.47$)$ \\
\hline \multicolumn{3}{|l|}{ Group } \\
\hline Non-diabetic & $7.8(246 / 3136)$ & 1 (reference) \\
\hline Screen-detected diabetes & $9.1(6 / 66)$ & $1.23(0.52$ to 2.88$)$ \\
\hline Self-reported diabetes & $12.8(17 / 133)$ & $1.78(1.05$ to 3.04$) \neq, p=0.032$ \\
\hline Type 1 diabetes & $6.7(7 / 104)$ & $0.86(0.39$ to 1.87$)$ \\
\hline \multirow[t]{3}{*}{ Type 2 diabetes } & $9.8(76 / 774)$ & $1.37(1.003$ to 1.89$) \neq, p=0.048$ \\
\hline & \multicolumn{2}{|c|}{ General population and patients with type 2 diabetes $(n=4109)$} \\
\hline & \multicolumn{2}{|l|}{ Diabetes $\dagger$} \\
\hline Explanatory variables & Percentage $\left(\mathrm{n} / \mathrm{n}_{\text {total }}\right)$ & Adjusted OR* with $95 \% \mathrm{Cl}$ \\
\hline \multicolumn{3}{|l|}{ Sex } \\
\hline Men & $29.8(586 / 1967)$ & 1 (reference) \\
\hline Women & $18.1(387 / 2142)$ & $0.56(0.46$ to 0.67$) \neq, p=0.001$ \\
\hline \multicolumn{3}{|l|}{ Filaggrin } \\
\hline Wild type & $23.2(874 / 3764)$ & 1 (reference) \\
\hline Null mutation & $28.2(99 / 345)$ & $1.50(1.10$ to 2.06$) \neq, p=0.011$ \\
\hline \multicolumn{3}{|l|}{ BMI $\left(\mathrm{kg} / \mathrm{m}^{2}\right)$} \\
\hline$<25$ & $9.7(167 / 1713)$ & 1 (reference) \\
\hline $25-30$ & $23.2(341 / 1469)$ & $1.97(1.56$ to 2.47$) \neq, p=0.001$ \\
\hline$>30$ & $49.1(461 / 905)$ & $7.36(5.79$ to 9.36$) \neq, p=0.001$ \\
\hline \multicolumn{3}{|c|}{$\begin{array}{l}\text { Non-diabetic, healthy controls from the general population in Copenhagen; Screen-detected diabetes, diabetes screening group defined as } \\
\text { subjects who did not report diabetes but who had a fasting blood glucose } \geq 7 \text { and/or glycated haemoglobin } \geq 6.5 \% \text {. Self-reported diabetes, } \\
\text { diabetes group defined as subjects who gave an affirmative answer to the question: 'Have you ever been told that you suffered from diabetes?' } \\
\text { ^Mutually adjusted for variables shown in the table and age. } \\
\text { †Diabetes was defined as belonging to the 'screen-detected diabetes group,' the 'self-reported diabetes group,' or the 'type } 2 \text { diabetes group.' } \\
\text { The 'type } 1 \text { diabetes groups' was regarded as missing data. } \\
\text { †Statistically significant. }\end{array}$} \\
\hline
\end{tabular}

$9.9 \%(95 \%$ CI 8.8 to 10.9$)$ in non-diabetics, $9.1 \%(95 \%$ CI 3.9 to 18.8$)$ and $9.1 \%(95 \%$ CI 2.1 to 16.1$)$ in screendetected diabetics, $12.8 \%(95 \%$ CI 8.0 to 19.6$)$ and $13.5 \%(95 \%$ CI 7.6 to 19.3$)$ in self-reported diabetics, $6.7 \%(95 \%$ CI 3.1 to 13.5$)$ in patients with type 1 diabetes and $9.8 \%(95 \%$ CI 7.9 to 12.1$)$ in patients with established type 2 diabetes. In a crude data analysis, no significant difference could be identified between nondiabetic participants from the general population and, respectively, participants from the general population with screen-detected diabetes $(p=0.71)$ or patients with type 1 diabetes $(\mathrm{p}=0.67)$. However, in patients with type 2 diabetes $(p=0.073)$, an almost significant association was detected, and in participants from the general population with self-reported diabetes, a significant association was identified $(p=0.04)$. Some $41.7 \%$ had a BMI below $25 \mathrm{~kg} / \mathrm{m}^{2} \quad($ men $=32.5 \%$, women $=50.6 \%)$, $35.8 \%$ had a BMI between 25 and $30 \mathrm{~kg} / \mathrm{m}^{2}$ $(\mathrm{men}=43.8 \%$, women $=28.8 \%)$, whereas $22 \%$ had a BMI above $30 \mathrm{~kg} / \mathrm{m}^{2}(\operatorname{men}=23.8 \%$, women $=20.7 \%)$.

A logistic regression model was performed with 'filaggrin null mutation status' as the dependent variable and revealed no significant interaction term $(p=0.33)$ between age and diabetes. This means that stratification by age group was not necessary. However, the analysis revealed a positive and significant association between filaggrin null mutation status and, respectively, 'selfreported diabetes' and 'type 2 diabetes,' when compared with non-diabetic controls from the general population. Another logistic regression analysis was performed with 'diabetes' as the dependent variable and revealed a positive and significant association with filaggrin null genotype (table 2). An interaction term between filaggrin mutation status and BMI was significant $(p=0.03)$; hence, filaggrin mutations were positively associated with diabetes in subjects with low BMI. Thus, when separate regression analyses were made with diabetes as the dependent variable and sex, age and filaggrin mutation as the explanatory variables in subjects with $\mathrm{BMI}<25$, $\mathrm{BMI}=25-30$ and $\mathrm{BMI}>30 \mathrm{~kg} / \mathrm{m}^{2}$, the $\mathrm{OR}$ for filaggrin mutation was, respectively, 2.08 (95\% CI 1.15 to 3.76$), 1.51$ (95\% CI 0.91 to 2.48 ) and 1.11 (95\% CI 0.66 to 1.88 ).

Finally, a logistic regression analysis restricted to the general population data was performed with 'atopic dermatitis' as the independent variable and revealed a positive and significant association between atopic 
Table 3 Logistic regression analyses with the outcome diabetes status and adjusted for variables shown in the table as well as age

General population only $(n=3335)$

Atopic dermatitis $^{18}$

Explanatory variables

Crude OR with 95\% Cl

Adjusted OR* with $95 \% \mathrm{Cl}$

Sex

Men

Women

Filaggrin

Wild type

Null mutation

Group

Non-diabetic

Screen-detected diabetes

Self-reported diabetes
1 (reference)

2.27 (1.79 to 2.95$) \dagger, p<0.001$

1 (reference)

2.71 (1.92 to 3.71 ) $\dagger, p<0.001$

1 (reference)

0.91 (0.39 to 2.12)

1.42 (0.85 to 2.37$)$
1 (reference)

2.25 (1.74 to 2.89 ) †, $p<0.001$

1 (reference)

2.65 (1.92 to 3.67$) \dagger, p<0.001$

1 (reference)

1.22 (0.51 to 2.89 )

1.72 (1.01 to 2.93$) \dagger, p=0.045$

Non-diabetic: healthy controls from the general population in Copenhagen; Screen-detected diabetes: diabetes screening group defined as subjects who did not report diabetes but who had a fasting blood glucose $\geq 7$ and/or glycated haemoglobin $\geq 6.5 \%$; Self-reported diabetes: diabetes group defined as subjects who gave an affirmative answer to the question: 'Have you ever been told that you suffered from diabetes?' *Mutually adjusted for variables shown in the table and age.

†Statistically significant.

dermatitis and self-reported diabetes (table 3). A test for interaction between diabetes subgroup and filaggrin mutation status was negative $(\mathrm{p}=0.88)$. Thus, the increased risk of diabetes in participants with atopic dermatitis did not depend on filaggrin mutation status.

\section{DISCUSSION}

Data from the general population study suggested that the prevalence of filaggrin null mutations and atopic dermatitis was higher in adult Danes who reported diabetes than in non-diabetics (tables 1-3). This finding was replicated when an independent sample of Danish patients with type 2 diabetes was filaggrin-genotyped and compared with participants from the general population who did not report diabetes and who had normal fasting plasma-glucose and HbAlc levels (table 2). No information about atopic dermatitis status was available from the sample of type 1 and 2 diabetes patients. For this reason, we could not determine whether filaggrin mutation status worked as a proxy for atopic dermatitis owing to the strong positive association between atopic dermatitis and filaggrin null mutation status or whether an association could be attributed to the filaggrin null genotype only. However, we showed that filaggrin null mutations did not increase the risk of diabetes in subjects with atopic dermatitis. We found a borderline significant interaction between filaggrin mutations and BMI in a logistic regression analysis with diabetes as the dependent variable. This finding suggests that if filaggrin mutations truly increase the risk of developing diabetes, this might mainly be a factor in subjects with a low BMI. Thus, in obese subjects, factors other than the filaggrin genotype are of greater importance.

The vast majority of participants who reported diabetes were suspected of suffering from type 2 diabetes rather than the less prevalent type 1 diabetes. In support of this notion, a higher HOMA-IR was identified in participants who reported diabetes (table 1). A data analysis revealed that the prevalence of filaggrin null mutations was lower in patients with type 1 diabetes. This came as no surprise, since previous studies have identified an inverse association between type 1 diabetes and atopic dermatitis explained by the Th1/Th2 dichotomy. Thus, a Danish case-control study showed that among children who developed type 1 diabetes, the incidence of atopic dermatitis was significantly lower than in the controls before the onset of type 1 diabetes $(\mathrm{OR}=0.49$; $95 \%$ CI 0.39 to 0.63$).{ }^{10}$ A large German case-control study showed that atopic dermatitis was less frequent in diabetic $(13.3 \%)$ than in non-diabetic children $(18.0 \%)$ and that atopic dermatitis was significantly associated with a reduced risk of type 1 diabetes (adjusted $\mathrm{OR}=0.71 ; 95 \%$ CI 0.53 to 0.96$){ }^{11}$

There were weaknesses in this study that should be addressed. First, genotyping was only performed for R501X and 2282del4, which cover approximately $85 \%$ of null mutations in the filaggrin gene among Caucasians. ${ }^{19}$ Second, the occurrence of atopic dermatitis was based on the UK Working Party's Criteria, which have a sensitivity and specificity of $92 \%$ and $81 \%$, respectively. ${ }^{18}$ Third, the question used to identify subjects with diabetes in the general population study has never been validated. Although this may have contributed additionally to misclassification in this study, table 1 shows that variables differed markedly between participants with and without self-reported diabetes. Missing information about serum C-peptid concentrations, insulin therapy and onset of diabetes might have been a better way to establish a diabetes diagnosis. Fourth, owing to small study populations, random error may have affected the study outcome. Thus, since this is the first study on this topic, we cannot exclude the possibility that the observed associations could be a type 1 error. Fifth, selection bias may have influenced the positive association between self-reported atopic dermatitis and type 2 diabetes, as patients who visit their physician because of a chronic disorder are more 
likely to undergo evaluation for other disorders. However, since patients with type 1 diabetes had a lower prevalence of filaggrin null mutations, and since such patients also regularly visit their physician, this may explain the positive association only to a small degree. Since the onset of atopic dermatitis occurs primarily in early childhood, and type 2 diabetes typically begins in adulthood, atopic dermatitis is likely to precede diabetes. Despite the presented weaknesses, general population studies are generally less biased than studies including patients and may be used to generate new hypotheses.

This study had a very novel finding and raises important questions, that is, is the increasing prevalence of type 2 diabetes and atopic dermatitis related? Could our findings be explained by an increased risk of diabetes following the use of topical corticosteroids in individuals with a disrupted skin barrier, despite such medicaments generally penetrating to a very small degree? Could repeated short-term oral corticosteroid therapy in some individuals with moderate to severe atopic dermatitis increase the risk of type 2 diabetes? Could chemicals, proteins and haptens that penetrate filaggrin-deficient skin more easily than normal skin in fact increase the propensity to develop low-grade inflammation, which again, in concert with other factors, could increase the risk of type 2 diabetes? These clinical questions are definitely important to explore further, since they may influence the diagnostic work-up and clinical course for patients with atopic dermatitis. Hence, further studies are obviously warranted to confirm or falsify our results, preferably prospective ones. A recent study is indirectly in favour of an association between atopic dermatitis and type 2 diabetes. ${ }^{20}$ In children with term births, maternal gestational diabetes was significantly associated with atopic dermatitis ( $\mathrm{OR}=7.2 ; 95 \%$ CI 1.5 to 34.5$)$ and allergen sensitisation ( $\mathrm{OR}=5.7 ; 95 \%$ CI 1.2 to 28.0$)$ in the offspring. If an association between atopic dermatitis/filaggrin null genotype and type 2 diabetes can be replicated in other studies, it might be considered to screen patients with atopic dermatitis for diabetes to a higher degree or limit the use of oral corticosteroid therapy. Since we believe this area should be explored further, we plan to reinvestigate the association between the filaggrin null mutations and diabetes in a 30-year follow-up study from the general population in Copenhagen and conduct cross-linkage studies of diabetes and atopic dermatitis databases.

\footnotetext{
Author affiliations:

${ }^{1}$ National Allergy Research Centre, Department of Dermato-Allergology, Copenhagen University Hospital Gentofte, Copenhagen, Denmark ${ }^{2}$ Research Centre for Prevention and Health, Glostrup Hospital, University of Copenhagen, Copenhagen, Denmark

${ }^{3}$ Hagedorn Research Institute and Steno Diabetes Center, Gentofte, Denmark ${ }^{4}$ Faculty of Health Sciences, University of Southern Denmark, Odense, Denmark

${ }^{5}$ Department of Clinical Experimental Research, Glostrup Research Institute, Glostrup Hospital, University of Copenhagen, Copenhagen, Denmark ${ }^{6}$ Faculty of Health Science, University of Aarhus, Aarhus, Denmark ${ }^{7}$ Faculty of Health Sciences, University of Copenhagen, Copenhagen, Denmark ${ }^{8}$ Department of Clinical Biochemistry, Copenhagen University Hospital Gentofte, Copenhagen, Denmark
}

Funding The Danish Board of Health, The Danish Environmental Protection Agency, The Copenhagen County Research Foundation, The Velux Foundation, Denmark and The Danish Scientific Research Council.

\section{Competing interests None.}

Patient consent Obtained.

Ethics approval Ethics approval was provided by the Ethical Committee of Copenhagen County (KA-20060011)

Provenance and peer review Not commissioned; externally peer reviewed.

Data sharing statement Data will not be publically accessible. Interested individuals may contact the authors.

\section{REFERENCES}

1. Candi E, Schmidt R, Melino G. The cornified envelope: a model of cell death in the skin. Nat Rev Mol Cell Biol 2005;6:328-40.

2. Palmer CN, Irvine AD, Terron-Kwiatkowski A, et al. Common loss-of-function variants of the epidermal barrier protein filaggrin are a major predisposing factor for atopic dermatitis. Nat Genet 2006;38:441-6.

3. Thyssen JP, Johansen JD, Linneberg A, et al. The association between null mutations in the filaggrin gene and contact sensitization to nickel and other chemicals in the general population. Br J Dermatol 2010;162:1278-85.

4. Smith FJ, Irvine AD, Terron-Kwiatkowski A, et al. Loss-of-function mutations in the gene encoding filaggrin cause ichthyosis vulgaris Nat Genet 2006;38:337-42.

5. Palmer CN, Ismail T, Lee SP, et al. Filaggrin null mutations are associated with increased asthma severity in children and young adults. J Allergy Clin Immunol 2007;120:64-8.

6. Weidinger $\mathrm{S}$, Illig $\mathrm{T}$, Baurecht $\mathrm{H}$, et al. Loss-of-function variations within the filaggrin gene predispose for atopic dermatitis with allergic sensitizations. J Allergy Clin Immunol 2006;118:214-19.

7. Sakai S, Endo Y, Ozawa N, et al. Characteristics of the epidermis and stratum corneum of hairless mice with experimentally induced diabetes mellitus. $J$ Invest Dermatol 2003;120:79-85.

8. Sakai S, Kikuchi K, Satoh J, et al. Functional properties of the stratum corneum in patients with diabetes mellitus: similarities to senile xerosis. Br J Dermatol 2005;153:319-23.

9. Seirafi $\mathrm{H}$, Farsinejad K, Firooz A, et al. Biophysical characteristics of skin in diabetes: a controlled study. J Eur Acad Dermatol Venereol 2009;23:146-9.

10. Olesen $A B$, Juul $\mathrm{S}$, Birkebaek $\mathrm{N}$, et al. Association between atopic dermatitis and insulin-dependent diabetes mellitus: a case-control study. Lancet 2001;357:1749-52.

11. Rosenbauer J, Herzig P, Giani G. Atopic eczema in early childhood could be protective against Type 1 diabetes. Diabetologia 2003;46:784-8.

12. Bønnelykke K, Pipper CB, Tavendale R, et al. Filaggrin gene variants and atopic diseases in early childhood assessed longitudinally from birth. Pediatr Allergy Immunol 2010;21:954-61.

13. Thyssen JP, Linneberg A, Menne T, et al. Contact allergy to allergens of the TRUE-test (panels 1 and 2) has decreased modestly in the general population. Br J Dermatol 2009;161:1124-9.

14. Eising S, Svensson J, Skogstrand K, et al. Type 1 diabetes risk analysis on dried blood spot samples from population-based newborns: design and feasibility of an unselected case-control study. Paediatr Perinat Epidemiol 2007;21:507-17.

15. Thyssen JP, Linneberg A, Menne T, et al. The prevalence and morbidity of sensitization to fragrance mix I in the general population. Br J Dermatol 2009;161:95-101.

16. Ambye L, Rasmussen S, Fenger M, et al. Studies of the Gly482Ser polymorphism of the peroxisome proliferator-activated receptor gamma coactivator 1alpha (PGC-1alpha) gene in Danish subjects with the metabolic syndrome. Diabetes Res Clin Pract 2005;67:175-9.

17. Matthews DR, Hosker JP, Rudenski AS, et al. Homeostasis model assessment: insulin resistance and beta-cell function from fasting plasma glucose and insulin concentrations in man. Diabetologia 1985:28:412-19.

18. Williams HC, Burney PG, Pembroke AC, et al. Working Party's Diagnostic Criteria for Atopic Dermatitis. III. Independent hospital validation. Br J Dermatol 1994;131:406-16.

19. O'Regan GM, Sandilands A, McLean WH, et al. Filaggrin in atopic dermatitis. J Allergy Clin Immunol 2008;122:689-93.

20. Kumar R, Ouyang F, Story RE, et al. Gestational diabetes, atopic dermatitis, and allergen sensitization in early childhood. $J$ Allergy Clin Immunol 2009;124:1031-8. 